\title{
Configuración morfológica de la superficie palatina y lingual de los caninos deciduos superiores e inferiores en un grupo de indígenas misak de Silvia (Cauca, Colombia): una mirada desde la antropología dental
}

\author{
Morphological configuration of the palatal and lingual surface of superior \\ and inferior deciduous canines in a group of native Misak people from \\ Silvia (Cauca, Colombia): A dental anthropology perspective \\ Yulieth Annie-García ${ }^{1}$, Fernando Gustín ${ }^{1}$, Catalina Quiñonez ${ }^{1}$, \\ Lina Sacanamboy ${ }^{1}$, Mery-Hann Torres ${ }^{1}$, Lina Triana ${ }^{1}$, Diana Valencia ${ }^{1}$, \\ Edwin Rojas ${ }^{1}$, Jaime Gómez ${ }^{1}$, John Díaz ${ }^{1}$ Freddy Moreno ${ }^{2} \bowtie$
}

${ }^{1}$ Escuela de Odontología de la Universidad del Valle, Cali, Colombia

(iD) (ID) (ID) (iD) (ID)

2 Departamento de Ciencias Básicas de la Salud de la Pontificia Universidad Javeriana, Cali, Colombia.

Facultad de Ciencias de la Salud, Pontificia Universidad Javeriana, Cali, Colombia. Calle 18 \# 118-250, Edificio Raúl Posada, 2 ํ piso. Teléfono: (57 2) 3218200, ext.: 8927. Correo electrónico: fmorenog@javerianacali.edu.co

Recibido: 4 de octubre del 2017 Aprobado: 5 de marzo del $2018 \quad$ Disponible en línea: 20 de abril del 2018

Cómo citar este artículo: García Y, Gustín F, Quiñonez C, Sacanamboy L, Torres M, Triana L et al. Superficie lingual de los caninos deciduos en un grupo de indígenas misak (Cauca, Colombia): una mirada desde la antropología dental. Rev Nac Odontol. 2018;14(27), enero 2018. doi: https://doi.org/10.16925/od.v14i27.2340

\section{Resumen}

Introducción: la caracterización de la morfología de los dientes deciduos y permanentes, a partir del estudio de los rasgos morfológicos dentales coronales (RMDC), ha sido de gran utilidad en el propósito de estimar los procesos microevolutivos de los grupos étnicos contemporáneos.

Objetivo: describir la configuración morfológica de la superficie palatina y lingual de los caninos deciduos superiores e inferiores en un grupo de indígenas misak desde la mirada de la antropología dental.

Materiales y método: estudio descriptivo transversal de la frecuencia, la variabilidad, el dimorfismo sexual, la simetría bilateral y la correspondencia de ocho RMDC de 60 modelos de estudio (37 femeninos y 23 masculinos) obtenidos de un grupo de indígenas misak de Silvia (Cauca, Colombia).

Resultados: se observó alta frecuencia de la cresta mesial canina (73,3\% superiores y 70,0\% inferiores), fosas palatinas $(67,5 \%)$, fosas linguales $(59,2 \%$ ) y cresta central (67,5\% superiores y $59,2 \%$ inferiores); bajas frecuencias de forma de pala (17,5\% superiores y 10,8\% en los inferiores), tubérculo dental (19,1\% superiores) y cresta distal accesoria (32,5\% superiores); asimismo, prácticamente ausencia de surco de interrupción (6,7\% superiores y 3,3\% inferiores), tubérculo dental (0,0\% inferiores) y cresta distal accesoria (6,6\% inferiores). No hubo dimorfismo sexual. Sí hubo simetría bilateral.

Conclusiones: la superficie palatina y lingual de los caninos deciduos superiores e inferiores configuró un patrón de dos crestas marginales (la mesial más pronunciada que la distal), una cresta central y dos fosas palatinas (linguales). Este patrón morfológico es característico en poblaciones de diferente origen étnico y que ocupan el mismo territorio en el suroccidente colombiano.

Palabras clave: antropología dental, caninos, dentición decidua, indígenas misak, morfología dental, rasgos morfológicos dentales coronales. 


\title{
Morphological configuration of the palatal and lingual surface of superior and inferior deciduous canines in a group of native Misak people from Silvia (Cauca, Colombia): A dental anthropology perspective
}

\begin{abstract}
Introduction: The morphology of deciduous and permanent teeth's characterization obtained from studying dental crown traits (DCT), has been very useful to estimate micro-evolutionary processes of contemporary ethnic groups.

Objective: To describe -from dental anthropology's perspective- the morphological configuration of the palatal and lingual surface of superior and inferior deciduous canines in a group of Misak native people.

Materials and method: A cross-sectional descriptive study of frequency, variability, sexual dimorphism, bilateral symmetry and correspondence of 8 DCt of 60 study models (37 female and 23 male) obtained from a group of native Misak people from Silvia (Cauca, Colombia).

Results: High frequency of canine mesial crest was observed ( $73,3 \%$ superior and $70 \%$ inferior), palatal fossae $(67,5 \%)$, lingual pits $(59,2 \%)$ and central ridge (67,5\% superior and $59,2 \%$ inferior); low shovel-shaped frequencies $(17,5 \%$ superior and 10,8\% inferior), dental tubercle (19,1\% superior), and accessory distal crest (32,5\% superior). Also, it was possible to observe practically no furrow interruption (6,7\% superior and $3,3 \%$ inferior), dental tuber $(0,0 \%$ inferior) and accessory distal crest (6,6\% inferior). There was no sexual dimorphism, bilateral symmetry was also observed.

Conclusions: The palatal and lingual surface of superior and inferior deciduous canines configured a pattern of two marginal ridges (the mesial one resulted to be more evident than the distal one), a central ridge and two palatal (lingual) pits. This morphological pattern is characteristic in populations of different ethnic origins that occupy the same territory in southwestern Colombia.

Keywords: dental anthropology, canines, deciduous dentition, dental morphology, dental crown traits (DCT), Misak native people.

\section{Configuração morfológica da superfície palatina e lingual dos caninos decíduos superiores e inferiores de um grupo de indígenas misak de Silvia (Cauca, Colômbia): uma perspectiva da antropologia dentária}

\section{Resumo}

Introdução: a caracterização da morfologia dos dentes decíduos e permanentes, a partir do estudo dos traços morfológicos dentários coronários (RMDC, sigla em espanhol), tem sido muito útil na estimativa dos processos microevolutivos dos grupos étnicos contemporâneos.

Objetivo: descrever a configuração morfológica da superfície palatina e lingual dos caninos decíduos superiores e inferiores de um grupo de indígenas misak na perspectiva da antropologia dentária.

Materiais e método: estudo descritivo transversal da frequência, a variabilidade, o dimorfismo sexual, a simetria bilateral e a correspondência de oito rmoc de 60 modelos de estudo (37 femininos e 23 masculinos) provenientes de um grupo de indígenas misak de Silvia (Cauca, Colômbia).

Resultados: observou-se alta frequência de crista mesial canina ( $73,3 \%$ superiores e $70,0 \%$ inferiores), fossas palatinas $(67,5 \%)$, fossas linguais $(59,2 \%$ ) e crista central (67,5\% superiores e $59,2 \%$ inferiores); baixas frequências em forma de pá (17,5\% superiores e 10,8\% inferiores), tubérculo dentário (19,1\% superiores) e crista distal acessória (32,5\% superiores); também, ausência quase completa de sulco de interrupção (6,7\% superiores e 3,3 \% inferiores), tubérculo dentário ( $0,0 \%$ inferiores) e crista distal acessória (6,6\% inferiores). Não houve dimorfismo sexual. Houve simetria bilateral.

Conclusões: a superfície palatina e lingual dos caninos decíduos superiores e inferiores estabeleceu um padrão de duas cristas marginais (a mesial mais pronunciada que a distal), uma crista central e duas fossas palatinas (linguais). Este padrão morfológico é característico em populações de diferentes origens étnicas que ocupam o mesmo território no sudoeste da Colômbia.

Palavras-chave: antropologia dentária, caninos, dentição decídua, morfologia dentária, traços morfológicos dentários coronários, indígenas misak. 


\section{Introducción}

Nosotros somos de aquí como nace un árbol... Cosmogonía misak

Los caninos son cuatro dientes deciduos y cuatro dientes permanentes - dos superiores y dos inferiores en cada dentición - que, en cuanto clase, se constituyen en la transición en cada hemiarcada de los dientes anteriores a los dientes posteriores. $\mathrm{Su}$ función es amortiguar las fuerzas horizontales excesivas y deletéreas generadas durante los movimientos laterales, a fin de proteger los dientes posteriores, acción que depende de su alta capacidad nociceptiva ante los estímulos sensitivos durante la acción de los músculos de la masticación. A este mecanismo de protección se le conoce en la literatura como "función canina" o "llave canina". En esta, al iniciar el movimiento, la superficie vestibular de los caninos inferiores comienza a deslizarse sobre la superficie palatina de los superiores para producir la desoclusión de los dientes posteriores contralaterales. Anatómicamente, la corona de los caninos superiores presenta la superficie vestibular en forma de rombo, y es el borde incisal agudo - conformado por las vertientes cuspídeas mesial y distal que confluyen en un vértice cuspídeo-y la región cervical redondeada. La superficie palatina presenta una elevación o cresta central que se extiende desde el cíngulo hasta el vértice cuspídeo, y dos crestas marginales (mesial y distal), las cuales configuran - junto con la cresta central- dos fosas palatinas: mesial y distal, respectivamente. Los caninos inferiores presentan una corona más larga, angosta y de menor relieve lingual con relación a la de los superiores. En la superficie lingual, las fosas mesial y distal son menos notables que en los superiores (de la misma forma que el cíngulo es menos pronunciado), lo cual favorece que las mitades mesial y distal de la corona sean más simétricas [1-5].

Esta descripción topográfica - con orientación clínica - es la que de forma tradicional ha sido empleada en las facultades y escuelas de odontología para enseñar la morfología de los dientes [6]; sin embargo, la antropología dental ha realizado un abordaje mucho más detallado a partir de la integración de diferentes áreas del conocimiento (antropología, odontología, biología, paleontología y paleopatología), con el propósito de observar, registrar y analizar las variaciones anatómicas de los dientes humanos con base en el estudio de los rasgos morfológicos dentales coronales y radiculares $[7,8]$.

En la literatura especializada, estos rasgos morfológicos han sido denominados indistintamente como "caracteres", "características", "variantes", "aspectos", "atributos", "polimorfismos" y, algunos de ellos — de forma errónea-, "anomalías de forma" [9], con el propósito de describir una serie de estructuras positivas (cúspides y crestas) o negativas (surcos y fosas) que, desde el punto de vista genético, tienen el potencial de estar o no presentes en un sitio anatómico específico (frecuencia), de diferente manera (variabilidad) y en uno o más miembros de un grupo poblacional. De allí que su expresión fenotípica se reconozca como discreta y discontinua. Hasta el momento existen poco más de 100 rasgos morfológicos dentales que han sido reconocidos en las cuatro clases de dientes humanos, lo cuales han resultado de gran utilidad en los contextos antropológico, forense y odontológico [10-12].

En el caso de los caninos, tanto deciduos como permanentes, se ha logrado observar, identificar, describir y analizar cerca de 15 rasgos morfológicos dentales coronales (RMDC) que han demostrado ser útiles, dado su considerable valor taxonómico, a fin de estimar las relaciones biológicas (similitudes y disimilitudes respecto a la bilateralidad, dimorfismo sexual y correspondencia de acuerdo con el arco y la dentición) entre grupos humanos primitivos y modernos con base en su proceso microevolutivo específico. Esto ha permitido generar una idea aproximada sobre los desplazamientos y los contactos que han dado lugar al poblamiento (distribución geográfica) y a la variación étnica (patrón étnico) de la humanidad [13]. Ocho de estos RMDC se encuentran de forma específica en la superficie palatina (caninos superiores) y lingual (caninos inferiores), deciduos y permanentes $[13,14]$. No obstante, el número de estudios que han descrito la morfología a partir de la frecuencia y la variabilidad de los RMDC de los caninos deciduos son escasos, en comparación con los caninos permanentes. Esta diferencia se ha asociado, inicialmente, al poco interés de los antropólogos por la dentición decidua (debido a la baja posibilidad de encontrar restos óseos y dentales de niños en los sitios arqueológicos), lo que ha llevado a que no se hayan consolidado sistemas universales de registro, observación y análisis. Pese a esto, estudios en 
poblaciones contemporáneas han sugerido la utilidad de los RMDC de dientes deciduos al momento de evaluar las relaciones biológicas de las poblaciones humanas $[14,15]$.

Por tanto, el objetivo de este estudio fue describir la configuración morfológica de la superficie palatina y lingual de los caninos temporales superiores e inferiores, bajo la mirada de la antropología dental, a fin de aportar información al conocimiento de los procesos macro y microevolutivos de la dentición decidua humana.

\section{Materiales y métodos}

\section{A. Población y muestra}

Este fue un estudio descriptivo transversal cuantitativo de la frecuencia, la variabilidad, el dimorfismo sexual, la simetría bilateral y la correspondencia de ocho RMDC de los caninos deciduos superiores e inferiores de 60 modelos de estudio (37 femeninos y 23 masculinos) obtenidos de un grupo de indígenas misak de Silvia (Cauca, Colombia) (véase la figura 1). Se incluyeron individuos colombianos, de padres y abuelos colombianos, autorreconocidos como integrantes del grupo étnico misak, y seleccionados por conveniencia a partir de una encuesta y un examen intraoral, para luego incluir aquellos que asintieron y consintieron participar en el estudio. Los individuos estaban en un rango de entre nueve y 12 años de edad, teniendo en cuenta que los caninos superiores e inferiores deciduos erupcionan a los 18 meses ( \pm tres meses) y se pierden - por recambio dental fisiológicoentre los 10 y los 11 años ( \pm nueve meses) [16].

Los sistemas de observación, registro y análisis de los ocho RMDC empleados en este estudio fueron el sistema Hanihara para forma de pala [17]; el Arizona State University Dental Anthropology System (Asudas) para surco de interrupción, cresta mesial canina y cresta distal accesoria [18]; Grine para tubérculo dental [19]; y Goyes et al. para cresta central, fosas palatinas y fosas linguales [13] (tabla 1).

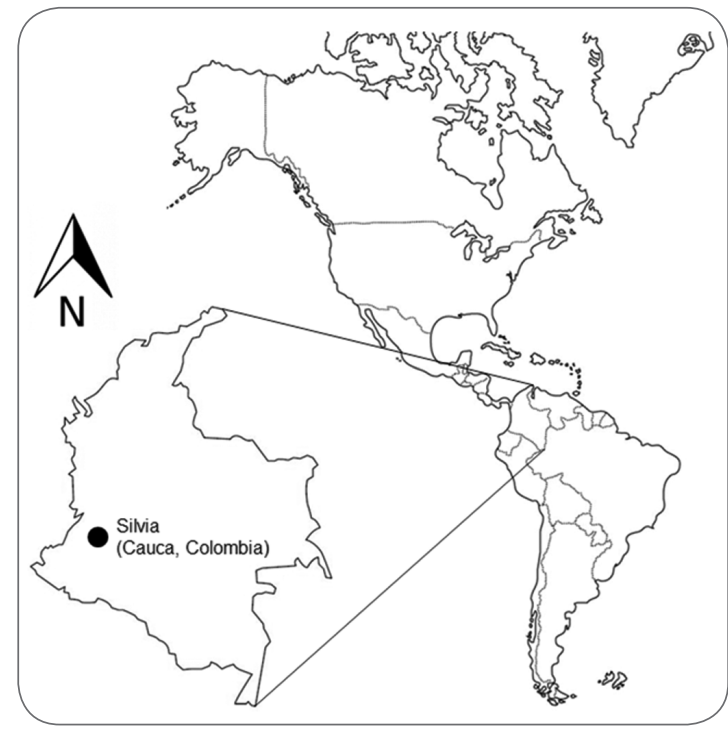

Figura 1. Localización geográfica del asentamiento del grupo étnico misak en Silvia (Cauca, Colombia)

Tabla 1. RMDC de caninos superiores e inferiores empleados en esta investigación

\begin{tabular}{|c|c|c|c|c|c|}
\hline \multirow{2}{*}{ Rasgo } & \multirow{2}{*}{ Diente } & \multirow{2}{*}{ Gradación } & \multicolumn{2}{|c|}{ Grados de expresión } & \multirow{2}{*}{$\begin{array}{l}\text { Método de } \\
\text { referencia }\end{array}$} \\
\hline & & & Rango & Presencia & \\
\hline Forma de pala & $53,63,73,83$ & $\begin{array}{l}\text { 0. Ausente } \\
\text { 1. Semipala } \\
\text { 2. Pala } \\
\text { 3. Pala marcado }\end{array}$ & $0-3$ & $2-6$ & Hanihara [17] \\
\hline Surco de interrupción & 53,63 & $\begin{array}{l}0 . \text { Ausente } \\
\text { 1. Mesolingual } \\
\text { 2. Distolingual } \\
\text { 3. Medial } \\
\text { 4. Mesolingual y } \\
\text { distolingual }\end{array}$ & $0-4$ & $1-4$ & Asudas [18] \\
\hline
\end{tabular}




\begin{tabular}{|c|c|c|c|c|c|}
\hline \multirow[t]{2}{*}{ Rasgo } & \multirow[t]{2}{*}{ Diente } & \multirow[t]{2}{*}{ Gradación } & \multicolumn{2}{|c|}{ Grados de expresión } & \multirow{2}{*}{$\begin{array}{l}\text { Método de } \\
\text { referencia }\end{array}$} \\
\hline & & & Rango & Presencia & \\
\hline Cresta mesial canina & 53,63 & $\begin{array}{l}\text { 0. Ausente } \\
\text { 1. Cresta } \mathrm{ML} \geq \mathrm{DL} \\
\text { 2. Cresta } \mathrm{ML}>\mathrm{DL} \\
\text { 3. Tipo de Morris }\end{array}$ & $0-3$ & $1-3$ & Asudas (18) \\
\hline Cresta distal accesoria & $53,63,73,83$ & $\begin{array}{l}0 . \text { Ausente } \\
\text { 1. Tenue } \\
\text { 2. Suave } \\
\text { 3. Moderado } \\
\text { 4. Fuerte }\end{array}$ & $0-4$ & $1-4$ & Asudas (18) \\
\hline Cresta central & $53,63,73,83$ & $\begin{array}{l}0 . \text { Ausente } \\
\text { 1. Presente }\end{array}$ & $0-1$ & 1 & Goyes et al. [13] \\
\hline Fosas palatinas & 73,83 & $\begin{array}{l}\text { 0. Ausentes } \\
\text { 1. Presente una sola } \\
\text { fosa } \\
\text { 2. Presentes ambas } \\
\text { fosas }\end{array}$ & $0-2$ & 1,2 & Goyes et al. [13] \\
\hline Fosas linguales & 73,83 & $\begin{array}{l}\text { 0. Ausentes } \\
\text { 1. Presente una sola } \\
\text { fosa } \\
\text { 2. Presentes ambas } \\
\text { fosas }\end{array}$ & $0-2$ & 1,2 & Goyes et al. [13] \\
\hline
\end{tabular}

Fuente: elaboración propia

\section{B. Estandarización del análisis morfológico}

Los observadores aprendieron a manejar los sistemas de registro y análisis de los ocho RMDC que se tuvieron en cuenta en este estudio, bajo protocolo de estandarización y doble enmascaramiento para controlar sesgos y lograr la unificación de los criterios de observación, de acuerdo con lo indicado por Nichol y Turner II [20]. La estimación del grado de concordancia se realizó mediante la prueba de Kappa, cuyos resultados -observadores mejor estandarizados - para los criterios de interobservador (observador vs. asesor) fueron del 92\% y del $95 \%$, e intraobservador (observador vs. observador) del $90 \%$ y del $94 \%$, respectivamente.

\section{Observación}

Una vez avalada la investigación por el Comité de Ética en Humanos de la Facultad de Salud de la Universidad del Valle, de acuerdo con la Resolución 008430 del Ministerio de la Protección Social [21] y con la Declaración de Helsinki [22], se procedió a la toma de impresiones con alginato Tropicalgin Zhermack $^{\circledR}$, obtención de modelos de estudio como yeso tipo III comercial americano y observación de los ocho RMDC con ayuda de una lupa de 10 aumentos, de un explorador de puntas finas y de las gradaciones de los sistemas empleados.

\section{Análisis estadístico}

Los datos obtenidos de la observación de los ocho RMDC se ingresaron en una plantilla en Excel ${ }^{\circ}$ y se procesaron en el software spss Versión 23.0 a través de pruebas estadísticas descriptivas para frecuencia, de análisis bivariado mediante pruebas no paramétricas (Wilcoxon y Mann-Whitney u) para dimorfismo sexual y simetría bilateral, y de análisis multivariado mediante coeficiente de correlación y concordancia (Spearman) para correlación entre rasgos. Una $\mathrm{p}<0,05$ se consideró estadísticamente significativa.

\section{Resultados}

Los RMDC de los caninos deciduos más frecuentes fueron la cresta mesial canina $(73,3 \%$ en los superiores y $70,0 \%$ en los inferiores), fosas palatinas $(67,5 \%)$, fosas linguales $(59,2 \%)$ y cresta central con la misma frecuencia que las fosas respectivas. Las 
frecuencias más bajas se encontraron en los rasgos forma de pala, tubérculo dental (superiores) y cresta distal accesoria (superiores); mientras que los rasgos surco de interrupción, tubérculo dental (inferiores) y cresta distal accesoria (inferiores) se encuentran prácticamente ausentes de la muestra (tabla 2). Ninguno de los rasgos presentó dimorfismo sexual $(\mathrm{p}<0,05)$ y todos los RMDC se expresaron de forma bilateral $(\mathrm{p}<0,05)$ (tabla 2$)$.

Hubo correlación entre los caninos superiores e inferiores para forma de pala (moderada $\mathrm{p}=0,056$ ), tubérculo dental (baja $\mathrm{p}=0,152)$ y cresta distal accesoria (nula $\mathrm{p}=0,000$ ). Al correlacionar la cresta central con los rasgos fosa palatina y fosa lingual, se encontraron correlaciones altas ( $\mathrm{p}=0,301$ y $\mathrm{p}=0,357$ respectivamente), de tal forma que la morfología de la superficie palatina y lingual de los caninos deciduos superiores e inferiores del grupo de indígenas misak incluidos en este estudio presentaron una tendencia a configurar dos fosas (mesial y distal) a partir de tres crestas (mesial, central y distal), en la que la mesial (cresta mesial canina) es más pronunciada que la distal (mesial y istal) (tabla 3).

Tabla 2. Frecuencias relativas de los rasgos morfológicos dentales

\begin{tabular}{|c|c|c|c|c|c|c|}
\hline \multirow{2}{*}{ Rasgos } & \multicolumn{2}{|c|}{ Frecuencia } & \multicolumn{2}{|c|}{ Dimorfismo sexual } & \multicolumn{2}{|c|}{ Bilateralidad } \\
\hline & Diente & $\%$ & Diente & Mann-Whitney $\mathrm{p}<0,05$ & Dientes & Wilcoxon $\mathrm{p}<0,05$ \\
\hline \multirow{4}{*}{$\begin{array}{l}\text { Forma de } \\
\text { pala }\end{array}$} & 53 & 16,7 & 53 & 0,217 & \multirow{2}{*}{$53-63$} & \multirow{2}{*}{0,739} \\
\hline & 63 & 18,3 & 63 & 0,536 & & \\
\hline & 73 & 10,0 & 73 & 0,083 & 73.83 & 0414 \\
\hline & 83 & 11,7 & 83 & 0,152 & /3-83 & 0,414 \\
\hline \multirow{2}{*}{ Surco de interrupción } & 53 & 6,7 & 53 & 0,580 & \multirow{2}{*}{$53-63$} & \multirow{2}{*}{0,317} \\
\hline & 63 & 3,3 & 63 & 0,287 & & \\
\hline \multirow{4}{*}{ Tubérculo dental } & 53 & 21,7 & 53 & 0,644 & \multirow{2}{*}{$53-63$} & \multirow{2}{*}{0,058} \\
\hline & 63 & 16,7 & 63 & 0,914 & & \\
\hline & 73 & 0,0 & 73 & 0,212 & \multirow{2}{*}{$73-83$} & \multirow{2}{*}{0,317} \\
\hline & 83 & 0,0 & 83 & 1,000 & & \\
\hline \multirow{4}{*}{ Cresta mesial canina } & 53 & 75,0 & 53 & 0,659 & \multirow{2}{*}{$53-63$} & \multirow{2}{*}{0,055} \\
\hline & 63 & 71,7 & 63 & 0,442 & & \\
\hline & 73 & 71,7 & 73 & 0,111 & \multirow{2}{*}{$73-83$} & \multirow{2}{*}{0,564} \\
\hline & 83 & 68,3 & 83 & 0,589 & & \\
\hline \multirow{4}{*}{ Cresta distal accesoria } & 53 & 31,7 & 53 & 0,591 & \multirow{2}{*}{$53-63$} & \multirow{2}{*}{0,822} \\
\hline & 63 & 33,3 & 63 & 0,728 & & \\
\hline & 73 & 5,0 & 73 & 0,729 & \multirow{2}{*}{$73-83$} & \multirow{2}{*}{0,454} \\
\hline & 83 & 8,3 & 83 & 0,670 & & \\
\hline \multirow{4}{*}{ Cresta central } & 53 & 68,3 & 53 & 0,224 & \multirow{2}{*}{$53-63$} & \multirow{2}{*}{0,780} \\
\hline & 63 & 66,7 & 63 & 0,662 & & \\
\hline & 73 & 65,0 & 73 & 0,334 & & \\
\hline & 83 & 61,7 & 83 & 0,946 & $73-83$ & 0,180 \\
\hline \multirow{2}{*}{ Fosas palatinas } & 53 & 73,3 & 53 & 0,291 & \multirow{2}{*}{$53-63$} & \multirow{2}{*}{1,000} \\
\hline & 63 & 78,3 & 63 & 0,486 & & \\
\hline \multirow{2}{*}{ Fosas linguales } & 73 & 68,3 & 73 & 0,798 & $73-83$ & 0.098 \\
\hline & 83 & 63,3 & 83 & 0,958 & $15-05$ & 0,090 \\
\hline
\end{tabular}


Tabla 3. Coeficiente de correlación de Spearman*

\begin{tabular}{|c|c|c|c|c|c|c|c|c|c|c|c|c|c|}
\hline & FPS & FPI & SI & TDS & TDI & CMS & CMI & CDAS & CDAI & CCS & CCI & FOPS & FOLI \\
\hline FPS & 1,000 & 0,579 & 0,221 & 0,164 & 0,173 & 0,422 & 0,116 & 0,183 & 0,002 & 0,393 & 0,068 & 0,470 & 0,028 \\
\hline FPI & & 1,000 & 0,215 & 0,043 & 0,126 & 0,426 & 0,075 & 0,024 & 0,036 & 0,413 & 0,086 & 0,474 & 0,114 \\
\hline SI & & & 1,000 & 0,188 & 0,034 & 0,008 & 0,227 & 0,028 & 0,143 & 0,006 & 0,178 & 0,019 & $-0,357$ \\
\hline TDS & & & & 1,000 & 0,222 & 0,210 & 0,044 & 0,387 & 0,186 & 0,147 & 0,065 & 0,120 & 0,162 \\
\hline TDI & & & & & 1,000 & 0,007 & 0,016 & 0,231 & 0,098 & 0,090 & 0,070 & 0,110 & 0,083 \\
\hline CMS & & & & & & 1,000 & 0,293 & 0,112 & 0,114 & 0,354 & 0,016 & 0,414 & 0,096 \\
\hline CMI & & & & & & & 1,000 & 0,071 & 0,060 & 0,082 & 0,162 & 0,047 & 0,257 \\
\hline CDAS & & & & & & & & 1,000 & 0,126 & 0,367 & 0,006 & 0,217 & 0,035 \\
\hline CDAI & & & & & & & & & 1,000 & 0,267 & 0,026 & 0,041 & 0,051 \\
\hline CCS & & & & & & & & & & 1,000 & 0,102 & 0,654 & 0,159 \\
\hline CCI & & & & & & & & & & & 1,000 & 0,053 & 0,728 \\
\hline FOPS & & & & & & & & & & & & 1,000 & 0,078 \\
\hline FOLI & & & & & & & & & & & & & 1,000 \\
\hline
\end{tabular}

${ }^{*}$ Interpretación correlación Rho de Spearman: $0=$ Nula; 0-0,2 = Muy baja; 0,2-0,4 = Baja; 0,4-0,6 = Moderada; 0,6-0,8 = Alta; 0,8-1 = Muy alta; 1 = Perfecta.

Convenciones: FPS = Forma de pala superiores; FPI = Forma de pala inferiores; sIS = Surco interrumpido; TDS = Tubérculo dental superiores; $\mathrm{TDI}=$ Tubérculo dental inferiores; $\mathrm{CMS}=$ Cresta mesial superiores; $\mathrm{CMI}=$ Cresta mesial inferiores; $\mathrm{CDAS}=$ Cresta distal accesoria superiores; $\mathrm{CDAI}=$ Cresta distal accesoria inferiores; $\mathrm{CCS}=$ Cresta central superiores; $\mathrm{CCI}=$ Cresta central inferiores; Fops $=$ Fosa palatina superiores; FOLI $=$ Fosa lingual inferiores.

Fuente: elaboración propia

\section{Discusión}

Debido a que el uso de los RMDC para caracterizar grupos étnicos se basa en el fuerte control genético, en la poca influencia medio ambiental, en los mínimos efectos del dimorfismo sexual y en la escasa dependencia biológica entre la expresión y la variabilidad de la morfología dental [14, 23], la discusión de los resultados se realiza con base en la frecuencia y la variabilidad, el dimorfismo sexual y la simetría bilateral, así como en la correlación entre rasgos.

\section{A. Frecuencia y variabilidad}

Si se tiene en cuenta que los RMDC que presentaron frecuencias significativas fueron la cresta mesial canina, las fosas palatinas y linguales y la cresta central, la configuración morfológica de la superficie palatina y lingual de los caninos deciduos correspondió, para el caso de los superiores, a la expresión de una cresta mesial más pronunciada que la distal, y a la presencia de una cresta central que configura dos fosas palatinas. En el caso de los inferiores, varía la expresión de la cresta mesial y de la cresta central, lo que configura uno o dos fosas linguales. De forma similar, altas frecuencias de cresta mesial canina fueron encontradas por Goyes et al. en un grupo de mestizos de Cali [13], y por Delgado [24, 25] en una muestra de afrodescendientes de Guapi. En contraste, este último autor encontró bajas frecuencias en muestras de afrodescendientes de Villarica, indígenas de Silvia y mestizos de Popayán.

De manera distintiva, el comportamiento de la cresta mesial canina o "canino de Bushman" ha sido uno de los RMDC más estudiado tanto en los caninos permanentes como deciduos. Su expresión describe de qué manera varía lo que en el contexto clínico odontológico se conoce como "reborde marginal mesopalatino" o "mesolingual", el cual traza su recorrido desde el tercio incisial — vertiente mesial del borde cuspídeo- hasta el tercio cervical [26, 27], región en la que se observó una baja expresión de tubérculo dental y prácticamente ausencia del surco de interrupción; de esta forma, en la región cervical palatina y lingual se describió un cíngulo romo de baja altura en el que se unen las crestas 
marginales mesial y distal, los cuales delimitan las fosas mesial y distal sin presentar surcos que se extiendan hasta unión amelocementaria. Goyes et al. encontraron frecuencias bajas del tubérculo dental y del surco de interrupción [13]. Datos muy similares a los reportados por Delgado [24, 25] en las muestras de Villarica, Silvia y Popayán. Caso contrario a la muestra de Guapi que presentó frecuencias medias.

Finalmente, la baja frecuencia de la forma de pala coincide con lo reportado en diferentes poblaciones asiáticas [28], de las cuales derivan todas las poblaciones indígenas pasadas y presentes -incluida la muestra de este estudio-, de acuerdo con los diferentes complejos dentales poblacionales que han sido diseñados mediante el estudio de las frecuencias de los RMDC. Sin embargo, es necesario tener en cuenta que estos complejos han sido elaborados fundamentalmente en la dentición permanente. En este sentido, Hanihara, en 1966 [29], planteó el complejo dental mongoloide con base en el estudio de la morfología de los dientes deciduos - tal como lo hizo con los dientes permanentes-y encontró que la simplificación morfológica progresiva a partir de las poblaciones del noreste asiático ha sido la constante en ambas denticiones, de tal forma que los dientes deciduos conservan características más primitivas que favorecen el estudio filogenético comparativo de las poblaciones humanas, por lo menos para el caso de los incisivos centrales superiores y los segundos molares superiores e inferiores. Pese a ello, la insuficiencia de estudios no ha permitido establecer patrones morfológicos en los dientes deciduos asociados a grupos étnicos específicos [30-34].

Tal como se ha discutido en otras publicaciones [24, 35-39], la región del suroccidente colombiano - de la cual proviene la muestra de este estudioha sufrido una serie de procesos históricos que favorecieron el mestizaje entre grupos de indígenas, de afrodescendientes y de mestizos caucasoides como consecuencia de los desplazamientos, las migraciones, el asentamiento y el aislamiento en el mismo territorio. Es por esto que, pese a la diversidad étnica y a la misma distribución geográfica, los dendogramas ubican en conglomerados próximos a diversas poblaciones del sur del departamento Valle del Cauca y del norte del departamento del Cauca al compartir la expresión de algunos RMDC. En el caso de los caninos deciduos, la frecuencia y la variabilidad de los RMDC que se tuvieron en cuenta en este estudio sugiere que la muestra de indígenas misak presenta niveles de mestizaje muy similares a las muestras de mestizos caucasoides, afrodescendientes e indígenas que han compartido históricamente el mismo territorio $[13,24,25]$.

\section{B. Dimorfismo sexual y simetría bilateral}

Dado que la odontogénesis de los dientes deciduos inicia de forma temprana durante el desarrollo embrionario, se podría pensar que por la presencia de dihidrostestosterona desde la séptima semana, la morfología de los dientes podría variar hacia expresiones más robustas de esmalte y dentina. Sin embargo, el dimorfismo sexual de los RMDC de los dientes deciduos y permanentes es prácticamente inexistente en las poblaciones humanas presentes [14]. Estos hallazgos contrastan con la evidencia que arrojan los estudios morfométricos debido, precisamente, a que los dientes caninos como clase han sido empleados con el fin de comprender la evolución del dimorfismo sexual dentro del desarrollo filogenético, ontogenético y socioecológico de los primates, al correlacionar los patrones intrasexuales de competencia, territorialidad y distribución de los recursos [40, 41].

En los primates, la reducción de tamaño y la simplificación de la morfología de los caninos ha ocurrido a expensas del sexo, de tal forma que los individuos masculinos tienden a tener dientes más grandes y más caracterizados. Este proceso, que empezó con la adquisición de la posición erecta, el andar bípedo y el uso de las manos para fabricar y emplear herramientas, ocasionó la reducción del biprognatismo facial y la microdoncia generalizada, sin afectar inicialmente la expresión morfológica (no obstante la gracilización de algunos RMDC). Esto puede asociarse a que la morfología de los caninos deciduos y permanentes ha presentado una alta preservación intraespecie, quizás por ser el único diente de su clase y contar con un tipo de diente, lo cual hace que tenga su propio campo morfogenético altamente conservado. Esta afirmación es igualmente respaldada por la alta simetría bilateral [41-45].

Respecto a la simetría bilateral, diferentes estudios han concluido que los RMDC de las dos hemiarcos son altamente conservados durante el proceso de morfogénesis dental, de tal forma que la posibilidad de presentar diferencias entre los dientes del lado derecho e izquierdo de una misma clase 
corresponde a un fenómeno aleatorio influenciado por el medio ambiente [46]. Tocheri [14] concluyó en su estudio que la morfología de la dentición decidua no presentaba dimorfismo sexual y contaba con simetría bilateral, respecto a la frecuencia relativa y la variabilidad de la expresión de los RMDC observados. En este estudio, ninguno de los RMDC estudiados en los caninos deciduos presentó dimorfismo sexual. El mismo resultado obtuvieron Goyes et al. (13) y Delgado [24, 25]. De esta forma, la información disponible sugiere que los RMDC de los caninos deciduos se encuentran, fundamentalmente, bajo control genético con influencia medioambiental poco significativa.

\section{Correlación entre rasgos}

Dada la gran similitud morfológica entre las clases de dientes, diferentes autores han estudiado la correlación de los RMDC entre la misma clase de dientes, pero de diferente tipo (p. ej. incisivo central superior e incisivo lateral superior); entre diferentes clases y diferentes tipos de dientes (p. ej. incisivo central superior y primer molar superior); entre la misma clase de dientes, pero diferente tipo (p. ej. primer molar superior y segundo molar superior); entre la misma clase de dientes, pero diferente arco (p. ej. primer molar superior y primer molar inferior); entre la misma clase de dientes, el mismo tipo y el mismo arco, pero diferente dentición (canino deciduo y canino permanente); además de otras combinaciones posibles reportadas en la literatura. Las conclusiones de estos estudios y la correlación positiva o negativa de la frecuencia y la variabilidad de la expresión de los diferentes RMDC se han asociado al proceso de morfogénesis dental de las cuatro clases de dientes [13, 23, 47, 48].

De esta forma, la teoría de los campos morfogéneticos de Butler y el modelo de los clones propuesto por Osborn han logrado explicar - con ciertas contradicciones- que las relaciones morfológicas que existen entre incisivos, caninos, premolares y molares provienen desde la odontogénesis a partir de las relaciones epiteliomesenquimáticas, de manera que la lámina dentaria se encuentra programada para formar las cuatro clases de dientes [7]. Butler, en 1939 [49], propuso que cada clase de diente (deciduos y permanentes) cuenta con un campo morfogenético propio, $y$ dentro del mismo existe un diente que cuenta con un gradiente de variación muy conservado y con una baja probabilidad de verse afectado por factores medioambientales. Así, el incisivo central es el diente gradiente del campo incisivo, el canino del campo canino y el primer molar del campo molar. Esta teoría también sugiere que los segundos y terceros dientes de cada clase, en la medida en que se alejan del diente gradiente, cuentan con la mayor variabilidad. Luego Dalhberg [50], en 1945, incluyó el campo premolar y combinó los campos de los dientes deciduos y permanentes. Si bien la mayoría de los estudios se han centrado en el campo molar a partir de las expresiones de los RMDC en el diente gradiente de ambas denticiones, se ha generalizado que los dientes deciduos al desarrollarse más temprano en la ontogenia tienen un nivel de conservación morfológica más alto que los permanentes, aun cuando ambas denticiones comparten la misma base genética. Es por esto que la variación de la morfología dental en un mismo individuo entendida como la variabilidad de los RMDC en sus diferentes grados de expresión-, puede atribuirse a la influencia del medio ambiente, por lo cual la variación se presentará en los dientes permanentes, cuyo desarrollo morfogenético es posnatal [47, $51,52]$. Al ser el canino un único diente dentro de su clase, en este estudio se realizó una correlación entre los RMDC que se tuvieron en cuenta, lo que permitió identificar la ausencia de forma en pala, de tubérculo dental y de cresta distal accesoria. El hecho más sobresaliente fue la alta correlación entre la expresión de la cresta central y las fosas palatina y lingual en los caninos deciduos superior e inferior, respectivamente, de tal forma que los caninos tienden a presentar una superficie palatina delimitada en su contorno proximal por las crestas marginales mesial (más pronunciada por la frecuencia del rasgo cresta mesial canina) y distal, las cuales confluyen, junto con la cresta central, hacia un cíngulo romo, mientras configuran dos fosas (la mesial y la distal) muy bien definidas. Esta misma caracterización de la superficie palatina fue descrita por Goyes et al., tanto en caninos deciduos como permanentes, por lo cual la evidencia disponible sugiere que el canino deciduo es el diente gradiente del campo morfogenético de la clase canina. Aun así, el canino permanente no ha presentado una variación considerable, pese a tener una mayor exposición a la influencia medio ambiental [13].

De esta forma, el estudio de la regulación genética y la variación fenotípica de la morfología dental a partir de los RMDC ha permitido hipotetizar sobre 
la microevolución de los diferentes grupos étnicos a través del tiempo, los cuales - a pesar de tener orígenes poblacionales diferentes- desarrollan patrones morfológicos similares al momento de compartir el mismo entorno geográfico biocultural y sufrir los mismos procesos de mestizaje histórico, lo cual ha resultado fundamental al momento de identificar desplazamientos y aislamientos. Sin embargo, tal como se ha discutido, las poblaciones americanas contemporáneas no han sido estudiadas de forma exahustiva, como sí se ha hecho en otras regiones del mundo. Es así como el grupo étnico misak comparte la expresión de ciertos RMDC en los caninos deciduos con otras poblaciones de mestizos caucasoides, indígenas y afrodescendientes que habitan la región del suroccidente colombiano, cuya frecuencia y variabilidad evidencia un comportamiento propio de grupos biológicamente mezclados, en el que se pueden observar características de poblaciones amerindias de origen sinodonte y africanas subsaharianas que, producto del mestizaje, generaron un mayor flujo de genes y una mayor variación biocultural $[13,23-25$, $53,54]$.

\section{Conclusiones}

El grupo de indígenas misak de Silvia (Cauca, Colombia) se caracterizó por presentar alta frecuencia de la cresta mesial canina, fosas palatinas (linguales) y cresta central; bajas frecuencias de forma de pala, tubérculo dental (superiores) y cresta distal accesoria (superiores); y ausencia de surco de interrupción, tubérculo dental (inferiores) y cresta distal accesoria (inferiores). De esta forma, la superficie palatina y lingual de los caninos deciduos superiores e inferiores configuró un patrón de dos crestas marginales (la mesial más pronunciada que la distal), una cresta central y dos fosas palatinas (linguales), la cual no presentó dimorfismo sexual y expresó simetría bilateral. Este mismo comportamiento se ha observado en otras poblaciones que comparten el mismo territorio geográfico pero presentan diferente origen étnico; por tanto, la frecuencia y la variabilidad de dichos RMDC puede asociarse al proceso histórico de mestizaje.

\section{Referencias}

[1] Kraus BS, Jordan RE, Abrams L. Anatomía dental y oclusión. México: Editorial Interamericana; 1972.

[2] Brand RW, Isselhard DE. Anatomía de las estructuras orofaciales. 6 ${ }^{\mathrm{a}}$ ed. Madrid: Harcourt Brace; 1999.

[3] Figún ME, Garino RR. Anatomía odontológica: funcional y aplicada. 2a ed. Chile: El Ateneo; 2002.

[4] Okeson JP. Tratamiento de oclusión y afecciones temporomandibulares. $7^{\mathrm{a}}$ ed. Buenos Aires: Elsevier; 2013.

[5] Nelson SJ. Anatomía dental, fisiología y oclusión de Wheeler. 10a ed. Madrid: Elsevier; 2015.

[6] Moreno S, Moreno F. Importancia clínica de la antropología dental. Rev Estomatol. 2007;15(2 Supl. 1):42-53.

[7] Scott GC, Turner II CG. The anthropology of modern human teeth: dental morphology and its variation in recent human populations. Londres: Cambridge University Press; 1997.

[8] Rodríguez JV. Dientes y diversidad humana: Avances de la antropología dental. Bogotá: Universidad Nacional de Colombia; 2003.

[9] King NM, Tongkoom S, Itthagarun A, Wong HM, Lee CK. A catalogue of anomalies and traits of the primary dentition of southern Chinese. J Clin Pediatr Dent. 2007;32(2):139-46. doi: https://doi. org/10.17796/jcpd.32.2.w76653r22rnnn713

[10] Scott GC, Turner II CG. Dental anthropology. Ann Rev Antrophol. 1998;17:99-126. doi: https://doi. org/10.1146/annurev.an.17.100188.000531

[11] Mayhall JT. Dental morphology: techniques and strategies. En: Biological anthropology of the human skeleton, katzenberg MA, Saunders SR (Eds). Nueva York: Willey-Liss; 2000. pp. 103-34.

[12] Rodríguez CD. La antropología dental y su importancia en el estudio de los grupos humanos. Rev Fac Odontol Univ Antioq. 2005;16(1 y 2):52-9.

[13] Goyes J, Guerrero L, Narváez N, Moreno F. Rasgos morfológicos dentales coronarios de caninos temporales y permanentes en un grupo de mestizos de Cali, Colombia. RCIO. 2011;2(5):1-13.

[14] Tocheri MW. The effects of sexual dimorphism, asymmetry and inter-trait association on the distribution of thirteen deciduous nonmetric traits in a sample of Pima Amerindians. Dent Anthropol. 2002;15(2-3):1-8. 
[15] Sciulli PW. Evolution of the dentition prehistoric Ohio Valley Native Americans: II. Morphology of the deciduous dentition. Am J Phys Anthropol. 1998;106(2):189-205. doi: https://doi.org/10.1002/ (SICI) $1096-8644$ (199806) 106:2<189::AID AJPA6>3.0.CO;2-L

[16] Schour I, Massler M. The development of the human dentition. JADA. 1941;28:1153-60.

[17] Hanihara K. Crown characters of the deciduous dentition of the Japanese-American hybrids. En: Brothwell DR (Ed.). Londres: Dental Anthropology. Pergamon Press; 1963.

[18] Turner II CG, Nichol CR, Scott GR. Scoring procedures for key morphological traits of the permanent dentition: the Arizona State University dental anthropology system. En: Nelly MA, Larsen CS (Eds). Advances in dental anthropology. Nueva York: Wiley-Liss; 1991.

[19] Grine F. Anthropological aspects of the deciduous teeth of South African blacks. En: Singer R, Lundy, J.K. (Eds). Variation, Culture and Evolution in African Populations. Johannesburgo: Witwatersrand University Press; 1986. pp. 47-83.

[20] Nichol CR, Turner II CG. Intra and inter-observer concordance in classifying dental morphology. Am J Phys Anthropol. 1986;69(3):299-315. doi: https:// doi.org/ 10.1002/ajpa.1330690303

[21] Colombia. Ministerio de Salud. Resolución por la cual se establecen las normas científicas, técnicas y administrativas para la investigación en salud. Resolución 008430/1993 del 4 de octubre. [Internet]. 1993. [citado 2017 may 9]. Disponible en: http://www.urosario.edu.co/urosario_files/a2/a24fb07af561-4fcc-b611-affff8554bb7.pdf

[22] Asociación Médica Mundial. Principios éticos para las investigaciones médicas en seres humanos, Declaración de Helsinki. [Internet]. 1964. [citado 2017 may 9]. Disponible en: http://www.urosario. edu.co/EMCS/Documentos/investigacion/declaracion_helsinki/

[23] Edgar HJH, Lease LR. Correlations between deciduous and permanent tooth morphology in a European American sample. Am J Phys Anthropol. 2007;133(1):726-34. doi: https://doi.org/10.1002/ ajpa. 20564

[24] Delgado-Burbano ME. Population affinities of African Colombians to Sub-Saharan Africans based on dental morphology. Homo. 2007;58(4):329-56. doi: https://doi.org/10.1016/j.jchb.2006.12.002

[25] Delgado-Burbano MD. Deciduous dental morphological diversity in contemporary Colombian ethnic groups. Dent Anthropol. 2008;21(2):33-45.
[26] Hillson S. Dental anthropology. Londres: Cambridge University Press; 1996. pp. 31-45.

[27] Irish JD, Morris DH. Technical note: canine mesial ridge (Bushman canine) dental trait definition. Am J Phys Anthropol.1996; 99(2):357-9. doi: https://doi. org/10.1002/ajpa.1330990202

[28] Kitagawa Y, Manabe Y, Oyamada J, Rokutanda A. Deciduous dental morphology of the prehistoric Jomon people of Japan: Comparison of nonmetric characters. Am J Phys Anthropol. 1995;97(2):10111. doi: https://doi.org/10.1002/ajpa.1330970203

[29] Hanihara K. Mongoloid dental complex in the deciduous dentition. J Anthropol Soc Nippon. 1966;74(2): 61-72. doi: https://doi.org/10.1537/ase1911. 74.61

[30] Hanihara K. Criteria for classification of crown characters of the human deciduous dentition. J Anthropol Soc Nippon. 1961;69:27-45. doi: https://doi. org/10.1537/ase1911.69.27

[31] Turner II CG. Advances in the dental search for native American origins. Acta Anthropogen. 1984;8:23-78.

[32] Zoubov AA. La antropología dental y la práctica forense. Maguaré. 1998;13:243-52.

[33] Irish JD. Characteristic high- and low- frequency dental traits in Sub-Saharan African populations. Am J Phys Anthropol. 1997;102(4):455-67. doi: https://doi.org/10.1002/(SICI)1096-8644(199704) 102:4<455::AID-AJPA3>3.0.CO;2-R

[34] Edgar HJH. Microevolution of African American dental Morphology. Am J Phys Anthropol. 2007;132:535-44. doi: https://doi.org/10.1002/ajpa 20550

[35] Moreno F, Moreno S, Díaz CA, Bustos EA. Prevalencia y variabilidad de ocho rasgos morfológicos dentales en jóvenes de tres colegios de Cali, 2002. Colomb Med. 2004;35(Supl 1):16-23.

[36] Aguirre LD, Castillo D, Solarte D, Moreno F. Frequency and variability of five non-metric dental crown traits in the primary and permanent dentitions of a racially mixed population from Cali, Colombia. Dent Anthropol. 2006;19(2):39-47.

[37] Rocha L, Rivas H, Moreno F. Frecuencia y variabilidad de la morfología dental en niños afro-colombianos de una institución educativa de Puerto Tejada, Cauca, Colombia. Colomb Med. 2007;38:210-21.

[38] Marcovich I, Prado E, Díaz P, Ortiz Y, Martínez C, Moreno F. Análisis de la morfología dental en escolares afro-colombianos de Villarica, Cauca, Colombia. Rev Fac Odontol Univ Antioq. 2012;24(1):37-61.

[39] Díaz E, García L, Fernández M, Palacio L, Ruiz D, Velandia N, et al. Frequency and variability of dental morphology in deciduous and permanent dentition of a Nasa indigenous group in the municipality of Morales, Cauca, Colombia. Colomb Med. 2014;45(1):15-24. 
[40] Plavcan JM, van Schaik CP, Kappeler PM. Competition, coalitions and canine size in primates. J Hum Evol. 1995;28(3):245-76. doi: https://doi.org/ 10.1006/jhev.1995.1019

[41] Simons EL, Plavcan JM, Fleagle JG. Canine sexual dimorphism in Egyptian Eocene anthropoid primates: Catopithecus and Proteopithecus. Proc Natl Acad Sci. 1999;96(5):2559-62. doi: https://doi. org/10.1073/pnas.96.5.2559

[42] Kinzey WG. Evolution of the human canine tooth. Am Anthropol. 1971;73(3): 680-694.

[43] Schwartz GT, Miller ER, Gunnell GF. Developmental processes and canine dimorphism in primate evolution. J Hum Evol. 2005;48(1):97-103. doi: https://doi.org/ 10.1016/j.jhevol.2004.10.005

[44] Greenfiel LO. Origin of the human canine: A new solution to an old enigma. Yearbook Phys Anthropol. 1992;35(Suppl 15):153-185. doi: https://doi. org/10.1002/ajpa.1330350607

[45] Schwartz GT, Dean C. Ontogeny of canine dimorphism in extant hominoids. Am J Phys Anthropol. 2001;115(3):269-283. doi: https://doi.org/10.1002/ ajpa.1081

[46] Mayhall JT, Saunders SR. Dimensional and discrete dental trait asymmetry relationships. Am J Phys Anthropol. 1986(3);69:403-11. doi: https://doi.org/ 10.1002/ajpa.1330690311

[47] Smith P, Koyoumonsky-Kaye E, Kaldaron W, Stern D. Directionality of dental trait frequency between human second deciduous and first permanent molars. Arch Oral Biol. 1987;32(1):5-9. doi: https://doi. org/10.1016/0003-9969(87)90146-4
[48] Ocampo AM, Sánchez JD, Martínez C, Moreno F. Correlación de diez rasgos morfológicos dentales coronales entre molares deciduos y permanentes en tres grupos étnicos colombianos. Rev Estomat. 2009;17(2):7-16.

[49] Butler PM. Studies of the mammalian dentition-differentiation of the post-canine dentition. Proc Zool Soc Lond B. 1939;109:1-36. doi: https://doi. org/10.1111/j.1469-7998.1939.tb00021.x

[50] Dahlberg AA. The changing dentition of man. J Am Dent Assoc. 1945;32:676-90. doi: https://doi. org/10.14219/jada.archive.1945.0112

[51] Butler PM. Comparison of the development of the second deciduous molar and first permanent molar in man. Arch Oral Biol. 1967;12:1245-60. doi: https://doi.org/10.1016/0003-9969(67)90126-4

[52] Butler PM. Ontogenetic aspects of dental evolution. Int J Dev Biol. 1995;39:25-34

[53] Heather JHE. Microevolution of African American dental morphology. Am J Phys Anthropol. 2007;132:535-44. doi: http://dx.doi.org/10.1002/ ajpa.20550

[54] Zúñiga S, Moreno S, Moreno F. Caracterización morfológica de los segundos molares temporales y los primeros molares permanentes de tres grupos étnicos de la región del Chocó (Colombia). Rev Nac Odontol. 2016;12(22):43-59. doi: http://dx.doi. org/10.16925/od.v12i22.1205 